\title{
ON UNIVERSALITY AND CONVERGENCE OF THE FOURIER SERIES OF FUNCTIONS IN THE DISC ALGEBRA.
}

\author{
C. PAPACHRISTODOULOS, M. PAPADIMITRAKIS.
}

\begin{abstract}
We construct functions in the disc algebra with pointwise universal Fourier series on sets which are $G_{\delta}$ and dense and at the same time with Fourier series whose set of divergence is of Hausdorff dimension zero. We also see that some classes of closed sets of measure zero do not accept uniformly universal Fourier series, although all such sets accept divergent Fourier series.
\end{abstract}

\section{INTRODUCTION AND NOTATION.}

Let $\mathbb{D}=\{z \in \mathbb{C}|| z \mid<1\}, \mathbb{T}=\{z \in \mathbb{C}|| z \mid=1\}=\mathbb{R} / 2 \pi$. We denote by $C(\mathbb{T})$ the set of complex continuous functions with the supremum norm $\|\cdot\|$ and, for $f \in C(\mathbb{T})$, by $S_{n}(f, t)$ the $n$-th partial sum of the Fourier series of $f$ at the point $t \in \mathbb{T}$,

$$
S_{n}(f, t)=\sum_{k=-n}^{n} \widehat{f}(k) e^{i k t},
$$

where

$$
\widehat{f}(k)=\frac{1}{2 \pi} \int_{-\pi}^{\pi} f(t) e^{-i k t} d t, \quad k \in \mathbb{Z},
$$

is the $k$-th Fourier coefficient of $f$.

Also, let $A(\mathbb{D})=\{f \in C(\mathbb{T}) \mid \widehat{f}(k)=0$ for $k<0\}$ be the disc algebra with the supremum norm.

If $(X, d)$ is a complete metric space, a property is said to be satisfied at quasi all points of $X$ if it is satisfied at a $G_{\delta}$ and dense set, i.e. at a topologically large set.

First we recall a few facts regarding the divergence of the partial sums $S_{n}(f, t)$ that the reader must have in mind.

2010 Mathematics Subject Classification. 47B35, 30H35, 30H10.

Key words and phrases. Universality, disc algebra.

The first author is supported by the project 3083-FOURIERDIG which is implemented under the "Aristeia II" Action of the "Operational Programme Education and Lifelong Learning" and is co-founded by the European Social Fund (ESF) and National Resources. 
The first, from [4], is that quasi all $f \in C(\mathbb{T})$ have the property that their Fourier series diverge at quasi all points of $\mathbb{T}$.

The second (see [3]) is the classical result that $E \subseteq \mathbb{T}$ is a set of divergence for $C(\mathbb{T})$, i.e. there is a continuous function whose Fourier series diverges at all points of $E$, if and only if $E$ is a set of infinite divergence for $C(\mathbb{T})$, i.e. there is a continuous function $f$ such that $\varlimsup\left|S_{n}(f, t)\right|=+\infty$ for all $t \in E$. Moreover, every set of Lebesgue measure zero is a set of divergence for $C(\mathbb{T})$.

It is not hard to see that a set $E \subseteq \mathbb{T}$ may be $G_{\delta}$ and dense and simultaneously of Lebesgue measure zero (see [9]). Of course, by Carleson's theorem a set of divergence for $C(\mathbb{T})$ has necessarily measure zero.

The third fact, from [1], is that the set $\left\{t|\varlimsup| \lim \left|S_{n}(f, t)\right|=+\infty\right\}$ has Hausdorff dimension equal to 1 for quasi all $f \in C(\mathbb{T})$.

Recently, in [7] and [2, a different notion of divergence has been studied. We present the definitions and the basic results of these papers.

Definition 1.1. Let $E \subseteq \mathbb{T}$. We say that $f \in C(\mathbb{T})$ is pointwise universal on $E$ if for every $g: E \rightarrow \mathbb{C}$ belonging to the Baire-1 class there exists a strictly increasing sequence of positive integers $\left(k_{n}\right)$ such that $S_{k_{n}}(f, t) \rightarrow g(t)$ for all $t \in E$.

$W e$ denote the class of these functions by $U_{p}(E)$.

Definition 1.2. Let $K \subseteq \mathbb{T}$ be a compact set. We say that $f \in C(\mathbb{T})$ is uniformly universal on $K$ if for every continuous $g: K \rightarrow \mathbb{C}$ there exists a strictly increasing sequence of positive integers $\left(k_{n}\right)$ such that $\left\|S_{k_{n}}(f, \cdot)-g\right\|_{K} \rightarrow 0$, where $\|\cdot\|_{K}$ is the supremum norm on $K$.

We denote the class of these functions by $U(K)$.

Of course the notions of pointwise universality and uniform universality coincide when the set $E=K$ is finite. In this case we speak about universality on $E=K$.

If we denote by $\mathcal{K}(\mathbb{T})$ the complete metric space of all nonempty compact subsets of $\mathbb{T}$ with the Hausdorff metric then we know that quasi all $f \in C(\mathbb{T})$ and quasi all $f \in A(\mathbb{D})$ are uniformly universal on quasi all sets $K \in \mathcal{K}(\mathbb{T})$. (For $C(\mathbb{T})$ see [7] and for $A(\mathbb{D})$ see [2].) And since quasi all sets in $\mathcal{K}(\mathbb{T})$ are perfect sets (see [6]) we know that there are perfect $K \subseteq \mathbb{T}$ such that $A(\mathbb{D}) \cap U(K) \neq \emptyset$.

Finally, we know that for each countable $E \subseteq \mathbb{T}$ quasi all $f \in C(\mathbb{T})$ and quasi all $f \in A(\mathbb{D})$ are pointwise universal on $E$. (For $C(\mathbb{T})$ see [7] and for $A(\mathbb{D})$ see [2].)

The proofs of the above results are not constructive. They use Baire's category theorem. Hence a first question which arises is to 
construct a uniformly or pointwise universal function. A second question is what can we say about the convergence of the Fourier series outside $E$ of a function in $U_{p}(E)$. If for example $f \in A(\mathbb{D}) \cap U_{p}(E)$, where $E$ is a countable dense set in $\mathbb{T}$, then the Fourier series of $f$ cannot converge at all points outside $E$, since $E \subseteq G \subseteq D$, where $G=\bigcap_{N=1}^{+\infty} \bigcup_{n=1}^{+\infty}\left\{t \mid S_{n}(f, t)>N\right\}$ and $D=\left\{t \mid S_{n}(f, t)\right.$ diverges $\}$, and $G$ is $G_{\delta}$ and dense and hence uncountable. Also, taking into account that the pointwise universal functions are highly divergent, we may ask whether it is possible that the above set $D$ has Hausdorff dimension less than 1 . We deal with these questions in sections 2 and 3. More precisely, we give a method to construct pointwise universal functions in $A(\mathbb{D})$ on finite and countably infinite sets. We also give a criterion for convergence of the Fourier series outside the set of pointwise universality and we see that the above set $D$ can even be of Hausdorff dimension equal to 0 . Of course, the above method can also be applied for functions in $C(\mathbb{T})$.

In section 4 we turn to the study of uniform universality (see definition 1.2). By Carleson's theorem the perfect sets which accept uniform universality must have Lebesgue measure zero. Of course the first class of such perfect sets that comes to mind consists of the familiar Cantor type sets. We prove that these sets do not accept uniform universality. Moreover, we prove that the same is true for a class of compact countable sets. This is in contrast with the well known fact that all sets of measure zero are sets of divergence for $C(\mathbb{T})$.

Finally, we close this paper with some open problems.

In the following the symbol $C$ will denote an absolute constant which may change from one relation to the next.

\section{Universality on finite $K \subseteq \mathbb{T}$ And CONVERgence on $\mathbb{T} \backslash K$.}

Let $N>n$. We consider the Fejer polynomials

$$
Q_{N, n}(t)=2 \sin N t \sum_{k=1}^{n} \frac{\sin k t}{k}=\sum_{m=-(N+n)}^{N+n} \widehat{Q_{N, n}}(m) e^{i m t},
$$

where

$$
\widehat{Q_{N, n}}( \pm m)= \begin{cases}\frac{1}{2 k}, & m=N-k, k=1, \ldots, n \\ -\frac{1}{2 k}, & m=N+k, k=1, \ldots, n \\ 0, & \text { otherwise }\end{cases}
$$

Obviously,

$$
Q_{N, n}(0)=0 .
$$


It is well known that the Fejer polynomials are uniformly bounded, i.e.

$$
\left\|Q_{N, n}\right\| \leq C
$$

Also,

$$
S_{N}\left(Q_{N, n}, 0\right)=\frac{1}{n}+\cdots+1 \sim \log n
$$

and the sequences of the Fourier coefficients of $Q_{N, n}$ are of uniform bounded variation, i.e.

$$
\sum_{m=-(N+n)}^{N+n+1}\left|\widehat{Q_{N, n}}(m-1)-\widehat{Q_{N, n}}(m)\right| \leq C .
$$

Finally, we have the estimate

$$
\left|S_{k}\left(Q_{N, n}, t\right)\right| \leq \frac{C}{|t|} \quad \text { for all } k \text { and } t \neq 0 .
$$

For all these properties see 3], Ch II, exercise 2.3.

Now, considering arbitrary $c \in \mathbb{C}$ and $\epsilon>0$ and setting

$$
P_{N, n}=\frac{c}{S_{N}\left(Q_{N, n}, 0\right)} Q_{N, n}
$$

it follows easily that if $N>n$ are large enough then

$$
\begin{gathered}
P_{N, n}(0)=0, \\
S_{N}\left(P_{N, n}, 0\right)=c, \\
\left\|P_{N, n}\right\|<\epsilon, \\
\sum_{m=-(N+n)}^{N+n+1}\left|\widehat{P_{N, n}}(m-1)-\widehat{P_{N, n}}(m)\right| \leq \epsilon, \\
\left|S_{k}\left(P_{N, n}, t\right)\right| \leq \frac{\epsilon}{|t|} \quad \text { for all } k \text { and } t \neq 0 .
\end{gathered}
$$

Let $\left\{c_{j} \mid j \in \mathbb{N}\right\}$ be a countable dense set in $\mathbb{C}$ and let $\epsilon, \epsilon_{j}>0$ with

$$
\epsilon=\sum_{j=1}^{+\infty} \epsilon_{j}
$$

Now for each $j$ we can choose arbitrarily large $N_{j}>n_{j}$ and polynomials $P_{N_{j}, n_{j}}$ such that

$$
\begin{gathered}
P_{N_{j}, n_{j}}(0)=0, \\
S_{N}\left(P_{N_{j}, n_{j}}, 0\right)=c_{j}, \\
\left\|P_{N_{j}, n_{j}}\right\|<\epsilon_{j}, \\
4
\end{gathered}
$$




$$
\sum_{m=-\left(N_{j}+n_{j}\right)}^{N_{j}+n_{j}+1}\left|\widehat{P_{N_{j}, n_{j}}}(m-1)-\widehat{P_{N_{j}, n_{j}}}(m)\right| \leq \epsilon_{j} .
$$

$$
\left|S_{k}\left(P_{N_{j}, n_{j}}, t\right)\right| \leq \frac{\epsilon_{j}}{|t|} \quad \text { for all } k \text { and } t \neq 0
$$

We also choose the $N_{j}>n_{j}$ to satisfy the inequalities

$$
2 N_{j}+\left(N_{j}+n_{j}\right)<2 N_{j+1}-\left(N_{j+1}+n_{j+1}\right)
$$

for all $j$ and we set

$$
\begin{aligned}
P_{j}(t) & =e^{2 i N_{j} t} P_{N_{j}, n_{j}}(t) \\
\mathcal{B}_{j} & =\left\{N_{j}-n_{j}, \ldots, 3 N_{j}+n_{j}\right\} \supseteq \operatorname{spectrum}\left(P_{j}\right) .
\end{aligned}
$$

From (6) we have

$$
\mathcal{B}_{j} \prec \mathcal{B}_{j+1},
$$

where $\prec$ means that the block $\mathcal{B}_{j}$ lies to the left of $\mathcal{B}_{j+1}$, that is $\max \mathcal{B}_{j}<\min \mathcal{B}_{j+1}$.

Hence (3) implies that the series

$$
\sum_{j=1}^{+\infty} P_{j}=g
$$

converges uniformly to a function $g \in C(\mathbb{T})$ such that

$$
\|g\|<\epsilon .
$$

Also, from (7) and (8) we get

$$
\widehat{g}(n)= \begin{cases}\widehat{P}_{j}(n)=\widehat{P_{N_{j}, n_{j}}}\left(n-2 N_{j}\right), & \text { if } n \in \mathcal{B}_{j}, j \in \mathbb{N} \\ 0, & \text { otherwise }\end{cases}
$$

In particular $\widehat{g}(n)=0$ for $n<0$ and thus $g$ belongs to $A(\mathbb{D})$.

Also, (1) and (2) imply

$$
S_{3 N_{j}}(g, 0)=c_{j} .
$$

Consequently, $g$ is universal on $K=\{0\}$.

Moreover, (4), (8) and (9) imply

$$
\sum_{n=0}^{+\infty}|\widehat{g}(n-1)-\widehat{g}(n)|<\epsilon
$$

i.e. the sequence $(\widehat{g}(n))$ is of bounded variation. Hence the Fourier series $\sum_{n=0}^{+\infty} \widehat{g}(n) e^{i n t}$ converges for $t \neq 0$ and uniformly in each closed 
interval of $\mathbb{T}$ which does not contain 0. See [10], Ch I, Theorem (2.6). Moreover, (5) implies

$$
\left|S_{k}\left(P_{j}, t\right)\right| \leq \frac{\epsilon_{j}}{|t|} \quad \text { for all } j, k \text { and } t \neq 0 .
$$

From (7) and (8) and the uniform convergence of the series $\sum_{j=1}^{+\infty} P_{j}$ we get

$$
S_{n}(g, t) \rightarrow g(t) \quad \text { as } n \rightarrow+\infty \text { and } n \notin \bigcup_{j=1}^{+\infty} \mathcal{B}_{j}
$$

The previous constructions can be extended for any finite number of points $t_{1}, \ldots, t_{m} \in \mathbb{T}$. For simplicity we present the construction for two points.

Let $t_{1} \neq t_{2}$ and $\left\{\left(a_{j}, b_{j}\right) \mid j \in \mathbb{N}\right\}$ be a countable dense set in $\mathbb{C}^{2}$. We consider the functions

$$
f_{1}(t)=g_{1}\left(t-t_{1}\right)=\sum_{j=1}^{+\infty} P_{j}^{(1)}(t), \quad f_{2}(t)=g_{2}\left(t-t_{2}\right)=\sum_{j=1}^{+\infty} P_{j}^{(2)}(t),
$$

where $g_{1}$ is the function $g$ constructed above with $\left(a_{j}\right)$ in place of $\left(c_{j}\right)$ and $g_{2}$ is the function $g$ with $\left(b_{j}\right)$ in place of $\left(c_{j}\right)$. Note that the polynomials $P_{j}^{(1)}, P_{j}^{(2)}$ have their spectrum in $\mathcal{B}_{j}$ and satisfy (11) with $t-t_{1}, t-t_{2}$ repsectively at the denominator of the right side.

Relation (10) becomes

$$
S_{3 N_{j}}\left(f_{1}, t_{1}\right)=a_{j}, \quad S_{3 N_{j}}\left(f_{2}, t_{2}\right)=b_{j} .
$$

Note that, since $N_{j}, n_{j}$ in the previous constructions can be chosen arbitrarily large, we may consider them to be the same for the functions $g_{1}, g_{2}$.

Then

$$
f_{1} \in A(\mathbb{D}) \cap U\left(\left\{t_{1}\right\}\right), \quad f_{2} \in A(\mathbb{D}) \cap U\left(\left\{t_{2}\right\}\right) .
$$

Also $S_{n}\left(f_{1}, t\right) \rightarrow f_{1}(t)$ for $t \neq t_{1}$ and $S_{n}\left(f_{2}, t\right) \rightarrow f_{2}(t)$ for $t \neq t_{2}$ and uniformly on every closed interval in $\mathbb{T}$ which does not contain $t_{1}, t_{2}$ and $S_{n}\left(f_{1}, t\right) \rightarrow f_{1}(t)$ and $S_{n}\left(f_{2}, t\right) \rightarrow f_{2}(t)$ for every $t$ as $n \rightarrow+\infty$ and $n \notin \bigcup_{j=1}^{+\infty} \mathcal{B}_{j}$.

Taking into account that the set $\left\{\left(a_{j}+f_{2}\left(t_{1}\right), b_{j}+f_{1}\left(t_{2}\right)\right)\right\}$ is dense in $\mathbb{C}^{2}$ we get the following for the function $f=f_{1}+f_{2}$ or, more generally, for the function $f=f_{1}+\cdots+f_{m}$ when $K=\left\{t_{1}, \ldots, t_{m}\right\}$.

Theorem 2.1. Let $K=\left\{t_{1}, \ldots, t_{m}\right\}$ be a finite set in $\mathbb{T}$. For every $\epsilon_{j}>0$ with $\sum_{j=1}^{+\infty} \epsilon_{j}<+\infty$ there are blocks $\mathcal{B}_{j}$ in $\mathbb{N}$ such that $\mathcal{B}_{1} \prec \mathcal{B}_{2} \prec$ ... and corresponding polynomials $P_{j}$ with $\operatorname{spectrum}\left(P_{j}\right) \subseteq \mathcal{B}_{j}$ and $\left\|P_{j}\right\|<\epsilon_{j}$ so that the function $f=\sum_{6}^{+\infty} P_{j}$ has $\operatorname{spectrum}(f) \subseteq \bigcup_{j=1}^{+\infty} \mathcal{B}_{j}$ 
and the following properties:

(i) $f \in A(\mathbb{D}) \cap U(K)$,

(ii) $\|f\| \leq \epsilon=\sum_{j=1}^{+\infty} \epsilon_{j}$,

(iii) $S_{n}(f, t) \rightarrow f(t)$ for every $t \notin K$ and uniformly on every closed interval in $\mathbb{T}$ which does not intersect $K$.

(iv) $\left|S_{k}\left(P_{j}, t\right)\right| \leq \epsilon_{j} \sum_{l=1}^{m} \frac{1}{\left|t-t_{l}\right|}$ for all $j, k$ and $t \neq t_{1}, \ldots, t_{m}$.

(v) $S_{n}(f, t) \rightarrow f(t)$ for every $t$ as $n \rightarrow+\infty$ and $n \notin \bigcup_{j=1}^{+\infty} \mathcal{B}_{j}$.

We note that the $\min \mathcal{B}_{j}$ can be taken arbitrarily large.

\section{Pointwise universality on Countably infinite $E \subseteq \mathbb{T}$ and CONVERGENCE ON $\mathbb{T} \backslash E$.}

Let $E=\left\{t_{l} \mid l \in \mathbb{N}\right\} \subseteq \mathbb{T}$ be a countably infinite set. We begin with the construction of a function $f \in A(\mathbb{D}) \cap U_{p}(E)$.

Let $E_{m}=\left\{t_{1}, \ldots, t_{m}\right\}$. By Theorem 2.1 we have that for each $\epsilon_{m, j}>$ 0 with $\sum_{j=1}^{+\infty} \epsilon_{m, j}<+\infty$ there are blocks $\mathcal{B}_{m, j}$ in $\mathbb{N}$ such that $\mathcal{B}_{m, 1} \prec$ $\mathcal{B}_{m, 2} \prec \ldots$ and corresponding polynomials $P_{m, j}$ with $\operatorname{spectrum}\left(P_{m, j}\right) \subseteq$ $\mathcal{B}_{m, j}$ and $\left\|P_{m, j}\right\|<\epsilon_{m, j}$ so that the function

$$
f_{m}=\sum_{j=1}^{+\infty} P_{m, j}
$$

belongs to $f \in A(\mathbb{D}) \cap U\left(E_{m}\right)$ and satisfies

$$
\left\|f_{m}\right\| \leq \epsilon_{m}=\sum_{j=1}^{+\infty} \epsilon_{m, j}
$$

and

$$
\left|S_{k}\left(P_{m, j}, t\right)\right| \leq \epsilon_{m, j} \sum_{l=1}^{m} \frac{1}{\left|t-t_{l}\right|} \quad \text { for all } k, j \text { and } t \neq t_{1}, \ldots, t_{m}
$$

Since $\min \mathcal{B}_{m, j}$ can be taken arbitrarily large, we may take $\mathcal{B}_{m, j}$ in the following diagonal order:

$$
\mathcal{B}_{1,1} \prec \mathcal{B}_{2,1} \prec \mathcal{B}_{1,2} \prec \mathcal{B}_{3,1} \prec \mathcal{B}_{2,2} \prec \mathcal{B}_{1,3} \prec \ldots
$$

We may also assume that

$$
\sum_{m=1}^{+\infty} \epsilon_{m}<+\infty
$$

We set

$$
f=\sum_{m=1}^{+\infty} f_{m}=\sum_{m=1}^{+\infty} \sum_{j=1}^{+\infty} P_{m, j}
$$


By (12), (14) it follows that $f \in A(\mathbb{D})$. We now prove that $f$ is pointwise universal on $E$.

Theorem 3.1. Let $f$ be the function constructed above.

(a) $f \in A(\mathbb{D}) \cap U_{p}(E)$.

(b) For each $t \in \mathbb{T} \backslash E$ satisfying the condition

$$
\max _{j} \epsilon_{m, j} \sum_{l=1}^{m} \frac{1}{\left|t-t_{l}\right|} \rightarrow 0
$$

we have

$$
S_{n}(f, t) \rightarrow f(t) .
$$

In particular, if $m \max _{j} \epsilon_{m, j} \rightarrow 0$ and the distance of $t$ from $E$ is positive, then $S_{n}(f, t) \rightarrow f(t)$.

Proof. (a) Let $h: E \rightarrow \mathbb{C}$ be an arbitrary function.

We first choose $m_{1}$ such that

$$
\sum_{m=m_{1}+1}^{+\infty} \epsilon_{m}<\frac{\delta_{1}}{3}
$$

where $\delta_{1}=1$.

From the fact that the blocks $\mathcal{B}_{m, j}$ are mutually disjoint and from Theorem 2.1, we get

$$
\begin{aligned}
S_{n}\left(f_{m}, t_{l}\right) \rightarrow f_{m}\left(t_{l}\right) \quad \text { as } n & \rightarrow+\infty, n \in \bigcup_{j=1}^{+\infty} \mathcal{B}_{m_{1}, j}, \\
& 1 \leq l \leq m_{1}, 1 \leq m \leq m_{1}-1 .
\end{aligned}
$$

From (12) it follows that

$$
\begin{aligned}
& \sum_{m=m_{1}+1}^{+\infty}\left|S_{n}\left(f_{m}, t_{l}\right)\right| \leq \sum_{m=m_{1}+1}^{+\infty} \sum_{j=1}^{+\infty} \epsilon_{m, j}<\frac{\delta_{1}}{3} \\
& 1 \leq l \leq m_{1}, n \in \bigcup_{j=1}^{+\infty} \mathcal{B}_{m_{1}, j} .
\end{aligned}
$$

Also, from the universality of $f_{m_{1}}$ on $E_{m_{1}}=\left\{t_{1}, \ldots, t_{m_{1}}\right\}$ and from (15) we get that there exists $n_{1} \in \bigcup_{j=1}^{+\infty} \mathcal{B}_{m_{1}, j}$ so that

$$
\left|S_{n_{1}}\left(f_{m_{1}}, t_{l}\right)-\left(h\left(t_{l}\right)-\sum_{m=1}^{m_{1}-1} f_{m}\left(t_{l}\right)\right)\right|<\frac{\delta_{1}}{3}, \quad 1 \leq l \leq m_{1}
$$




$$
\begin{aligned}
\left|S_{n_{1}}\left(f_{m}, t_{l}\right)-f_{m}\left(t_{l}\right)\right|< & \frac{\delta_{1}}{3\left(m_{1}-1\right)}, \\
& 1 \leq l \leq m_{1}, 1 \leq m \leq m_{1}-1 .
\end{aligned}
$$

Now we observe that the $n_{1}$-th Fourier sum of $f$ is a finite sum of $n_{1}$-th Fourier sums of the functions $f_{1}, f_{2}, \ldots, f_{m_{1}^{\prime}}$ for some $m_{1}^{\prime} \geq m_{1}$. Hence

$$
\begin{aligned}
S_{n_{1}}\left(f, t_{l}\right)- & h\left(t_{l}\right) \\
= & \left(S_{n_{1}}\left(f_{1}, t_{l}\right)-f_{1}\left(t_{l}\right)\right)+\cdots+\left(S_{n_{1}}\left(f_{m_{1}-1}, t_{l}\right)-f_{m_{1}-1}\left(t_{l}\right)\right) \\
& +\left(S_{n_{1}}\left(f_{m_{1}}, t_{l}\right)-\left(h\left(t_{l}\right)-\sum_{m=1}^{m_{1}-1} f_{m}\left(t_{l}\right)\right)\right) \\
& +S_{n_{1}}\left(f_{m_{1}+1}, t_{l}\right)+\cdots+S_{n_{1}}\left(f_{m_{1}^{\prime}}, t_{l}\right) .
\end{aligned}
$$

Finally, from (17), (18), (19) we get

$$
\left|S_{n_{1}}\left(f, t_{l}\right)-h\left(t_{l}\right)\right|<\delta_{1}, \quad 1 \leq l \leq m_{1} .
$$

Similarly, for $\delta_{2}=\frac{1}{2}$ there exists $m_{2}>m_{1}$ such that $\sum_{m=m_{2}+1}^{+\infty} \epsilon_{m}<$ $\frac{\delta_{2}}{3}$ and there exists $n_{2}>n_{1}, n_{2} \in \bigcup_{j=1}^{+\infty} \mathcal{B}_{m_{2}, j}$ such that

$$
\left|S_{n_{2}}\left(f, t_{l}\right)-h\left(t_{l}\right)\right|<\delta_{2}, \quad 1 \leq l \leq m_{2} .
$$

Continuing in this manner, we construct strictly increasing sequences of positive integers $\left(m_{N}\right),\left(n_{N}\right)$ such that

$$
\left|S_{n_{N}}\left(f, t_{l}\right)-h\left(t_{l}\right)\right|<\delta_{N}=\frac{1}{N}, \quad 1 \leq l \leq m_{N} .
$$

This implies that

$$
S_{n_{N}}(f, t) \rightarrow h(t), \quad t \in E
$$

and the proof of pointwise universality is complete.

(b) By our diagonal ordering of the blocks $\mathcal{B}_{m, j}$ every $n \in \mathbb{N}$ lies in some diagonal $\mathcal{B}_{k, 1}, \mathcal{B}_{k-1,2}, \ldots, \mathcal{B}_{1, k}$. I.e. $\max \mathcal{B}_{1, k-1}<n \leq \max \mathcal{B}_{k, 1}$ or $\max \mathcal{B}_{k-j+1, j}<n \leq \max \mathcal{B}_{k-j, j+1}$ for some $j=1,2, \ldots, k-1$.

Hence by the definition of $f$ we have

$$
\begin{aligned}
S_{n}(f, t)= & P_{1,1}(t)+P_{2,1}(t)+P_{1,2}(t)+\cdots \\
& +P_{k, 1}(t)+P_{k-1,2}(t)+\cdots+P_{k-j+1, j}(t)+S_{n}\left(P_{k-j, j+1}, t\right)
\end{aligned}
$$

if $n \in \mathcal{B}_{k-j, j+1}$. Note that the final term in the last sum is missing in case $\max \mathcal{B}_{k-j+1, j} \leq n<\min \mathcal{B}_{k-j, j+1}$.

Similarly, if $m>n$ then $m$ lies in some diagonal $\mathcal{B}_{p, 1}, \mathcal{B}_{p-1,2}, \ldots, \mathcal{B}_{1, p}$ with $p \geq k$. 
Therefore,

$$
\begin{aligned}
\left|S_{n}(f, t)-S_{m}(f, t)\right| \leq & \left|P_{k-j, j+1}(t)-S_{n}\left(P_{k-j, j+1}, t\right)\right| \\
& +\left(\left|P_{k-j-1, j+2}(t)\right|+\cdots+\left|P_{p-q+1, q}(t)\right|\right) \\
& +\left|S_{m}\left(P_{p-q, q+1}, t\right)\right|
\end{aligned}
$$

Now (13) implies

$$
\left|S_{m}\left(P_{p-q, q+1}, t\right)\right| \leq \epsilon_{p-q, q+1} \sum_{l=1}^{p-q} \frac{1}{\left|t-t_{l}\right|}
$$

and, together with (12),

$$
\left|P_{k-j, j+1}(t)-S_{n}\left(P_{k-j, j+1}, t\right)\right| \leq \epsilon_{k-j, j+1}+\epsilon_{k-j, j+1} \sum_{l=1}^{k-j} \frac{1}{\left|t-t_{l}\right|} .
$$

Hence

$$
\begin{aligned}
\left|S_{n}(f, t)-S_{m}(f, t)\right| \leq & \epsilon_{k-j, j+1}+\epsilon_{k-j, j+1} \sum_{l=1}^{k-j} \frac{1}{\left|t-t_{l}\right|} \\
& +\epsilon_{k-j-1, j+2}+\cdots+\epsilon_{p-q+1, q} \\
& +\epsilon_{p-q, q+1} \sum_{l=1}^{p-q} \frac{1}{\left|t-t_{l}\right|}
\end{aligned}
$$

If $n, m \rightarrow+\infty$ then $k, p \rightarrow+\infty$ and since the double series $\sum_{r, s} \epsilon_{r, s}$ converges, we get $\epsilon_{k-j, j+1}+\epsilon_{k-j-1, j+2}+\cdots+\epsilon_{p-q+1, q} \rightarrow 0$.

Regarding the term $r=\epsilon_{k-j, j+1} \sum_{l=1}^{k-j} \frac{1}{\left|t-t_{l}\right|}$ we have two cases. If $k-j$ is bounded then $j+1 \rightarrow+\infty$ and thus $r \rightarrow 0$. If $k-j \rightarrow+\infty$ then (15) implies $r \rightarrow 0$. Similarly, $\epsilon_{p-q, q+1} \sum_{l=1}^{p-q} \frac{1}{\left|t-t_{l}\right|} \rightarrow 0$.

We conclude that $\left(S_{n}(f, t)\right)$ converges. The proof will be complete if we show that $S_{n}(f, t) \rightarrow f(t)$ when $n \rightarrow+\infty$ and does not belong to any of the blocks $\mathcal{B}_{m, j}$. Indeed, in this case $(v)$ of Theorem 2.1 implies

$$
S_{n}(f, t)=\sum_{m=1}^{+\infty} S_{n}\left(f_{m}, t\right) \rightarrow \sum_{m=1}^{+\infty} f_{m}(t)=f(t)
$$

due to the uniform bound $\sum_{m=1}^{+\infty}\left|S_{n}\left(f_{m}, t\right)\right| \leq \sum_{m=1}^{+\infty} \epsilon_{m}$.

As an application we get the following for the function $f$ of Theorem 3.1 .

Theorem 3.2. Let $\left(\delta_{m}\right)$ be a decreasing sequence of positive numbers such that $\sum_{m=1}^{+\infty} \delta_{m}^{a}<+\infty$ for all a $>0$. Now, if $\max _{j} \epsilon_{m, j} \sum_{l=1}^{m} \frac{1}{\delta_{l}} \rightarrow 0$, then we have $S_{n}(f, t) \rightarrow f(t)$ outside a set of Hausdorff dimension zero. 
Proof. We consider

$$
I_{m}=\left(t_{m}-\delta_{m}, t_{m}+\delta_{m}\right), \quad D=\bigcap_{m=1}^{+\infty} \bigcup_{l=m}^{+\infty} I_{l} .
$$

Since $\sum_{m=1}^{+\infty} \delta_{m}^{a}<+\infty$ for all $a>0$, the set $D$ is of Hausdorff dimension zero.

Now, if $t$ is not in the countable set $E$ neither in $D$, then it satisfies (15). Indeed, let $t \notin E$ and $t \notin \bigcup_{l=m_{0}}^{+\infty} I_{l}$ for some $m_{0}$. Then if $m \geq m_{0}$ we have

$$
\max _{j} \epsilon_{m, j} \sum_{l=1}^{m} \frac{1}{\left|t-t_{l}\right|} \leq \max _{j} \epsilon_{m, j} \sum_{l=1}^{m_{0}-1} \frac{1}{\left|t-t_{l}\right|}+\max _{j} \epsilon_{m, j} \sum_{l=m_{0}}^{m} \frac{1}{\left|t-t_{l}\right|} .
$$

The first term of the right side obviously tends to 0 as $m \rightarrow+\infty$. As for the second term we have

$$
\max _{j} \epsilon_{m, j} \sum_{l=m_{0}}^{m} \frac{1}{\left|t-t_{l}\right|} \leq \max _{j} \epsilon_{m, j} \sum_{l=m_{0}}^{m} \frac{1}{\delta_{l}} \leq \max _{j} \epsilon_{m, j} \sum_{l=1}^{m} \frac{1}{\delta_{l}}
$$

which by our hypothesis tends to 0 as $m \rightarrow+\infty$.

Note that, if $E$ is dense in $\mathbb{T}$, the set of divergence of $S_{n}(f, t)$ is necessarily $G_{\delta}$ and dense and hence uncountable, although by the proper choice of $f$ it can be of Hausdorff dimension zero.

\section{Subsets of $\mathbb{T}$ NOT ACCEPting Uniform Universality.}

Let $K$ be a compact subset of $\mathbb{T}, K \neq \mathbb{T}$. In Theorem 2.2 of $[5$ and in [8] it was shown that $H(\mathbb{D}) \cap U(K)$ is a dense- $G_{\delta}$ subset of $H(\mathbb{D})$ when the latter has the topology of uniform convergence on compacta.

We now prove a special case of Theorem 4.3 of [5] replacing the condition appearing there with a much simpler one (number (20) in what follows).

Proposition 4.1. (a) Let $K$ be a compact proper subset of $\mathbb{T}$ and $t_{0} \in K$ with the following property:

for each infinite $\mathbb{M} \subseteq \mathbb{N}$ there are $a, b$ with $0<a<b<2 \pi$

$$
\text { and }[a / m, b / m] \cap\left(K-t_{0}\right) \neq \emptyset \text { for infinitely many } m \in \mathbb{M} \text {. }
$$

If $f \in H(\mathbb{D}) \cap U(K)$, then the Taylor series of $f$ is not $(C, 1)$ summable at $t_{0}$.

(b) Now let $K$ and $t_{0}$ be as in (a) and moreover let $K$ be symmetric with respect to $t_{0}$, i.e. $2 t_{0}-t \in K$ for every $t \in K$.

If $f \in L^{1}(\mathbb{T}) \cap U(K)$, then the Fourier series of $f$ is not $(C, 1)$ summable at $t_{0}$. 
Proof. (a) We denote by $\sum_{k=0}^{+\infty} c_{k} z^{k}$ the Taylor series of $f$ and by $S_{n}(f, t)=\sum_{k=0}^{n} c_{k} e^{i k t}$ the partial sums on $\mathbb{T}$ and let us assume that the Taylor series is $(\mathrm{C}, 1)$ summable at $t_{0}$.

Since $f \in U(K)$, for every $h \in C(K)$ there is a subsequence $S_{n_{j}}(f, t)$ which converges to $h$ uniformly on $K$. Property (20) implies that there is a subsequence of $\left(n_{j}\right)$, which without loss of generality we may assume equal to $\left(n_{j}\right)$, and corresponding $\theta_{j} \in K-t_{0}$ such that $a \leq n_{j} \theta_{j} \leq b$ for all $j$.

Then Theorem (12.16) of [10, Vol I, Ch III implies

$$
S_{n_{j}}\left(f, t_{0}+\theta_{j}\right)-\left(S_{n_{j}}\left(f, t_{0}\right)-s\right) e^{i n_{j} \theta_{j}} \rightarrow s,
$$

where $s$ is the $(\mathrm{C}, 1)$ sum of the Taylor series of $f$ at $t_{0}$.

Taking a further subsequence of $n_{j}$, we may assume that $n_{j} \theta_{j} \rightarrow \phi$ for some $\phi \in[a, b]$. Now the uniform convergence $S_{n_{j}}(f, t) \rightarrow h(t)$ on $K$ implies

$$
h\left(t_{0}\right)-\left(h\left(t_{0}\right)-s\right) e^{i \phi}=s .
$$

Choosing $h\left(t_{0}\right) \neq s$ we arrive at a contradiction.

(b) The proof is the same as before. If $S_{n}(f, t)=\sum_{k=-n}^{n} \widehat{f}(k) e^{i k t}$ are the partial sums of the Fourier series of $f$, we assume that their $(\mathrm{C}, 1)$ means at $t_{0}$ converge to some $s$. We consider $h \in C(K)$, the sequence $S_{n_{j}}(f, t)$ converging to $h$ uniformly on $K$ and the corresponding $\theta_{j}$ so that $\pm \theta_{j} \in K-t_{0}$ and $a \leq n_{j} \theta_{j} \leq b$ for all $j$.

We now apply Theorem (12.9) of [10], Vol I, Ch III and get

$$
\frac{1}{2}\left(S_{n_{j}}\left(f, t_{0}+\theta_{j}\right)+S_{n_{j}}\left(f, t_{0}-\theta_{j}\right)\right)-\left(S_{n_{j}}\left(f, t_{0}\right)-s\right) \cos n_{j} \theta_{j} \rightarrow s .
$$

Exactly as before we get $h\left(t_{0}\right)-\left(h\left(t_{0}\right)-s\right) \cos \phi=s$ and we arrive at a contradiction choosing $h\left(t_{0}\right) \neq s$.

We note that property (20) can be stated with "left hand" intervals $\left[t_{0}-(b / m), t_{0}-(a / m)\right]$ and the result of Proposition 4.1(a) remains unchanged.

Corollary 4.1. If $K$ is a compact proper subset of $\mathbb{T}$ which satifies property (20) for some $t_{0} \in K$ then $A(\mathbb{D}) \cap U(K)=\emptyset$. If moreover $K$ is symmetric with respect to $t_{0}$ then $C(\mathbb{T}) \cap U(K)=\emptyset$.

In particular the one third Cantor set $C \subseteq[0, \pi]$ satisfies property (20) for $t_{0}=0 \in C$ and hence $A(\mathbb{D}) \cap U(C)=\emptyset$. For the symmetric Cantor set $C^{*}=C \cup(-C) \subseteq[-\pi, \pi]$ we have that $C(\mathbb{T}) \cap U\left(C^{*}\right)=\emptyset$.

Proof. Since the Fourier series of any $f \in A(\mathbb{D})$ is $(\mathrm{C}, 1)$ summable at every point of $\mathbb{T}$, the first statement is obvious. 
Regarding the Cantor set $C$ and $t_{0}=0 \in C$ we shall prove a stronger version of property (20): if $0<a<b<2 \pi$ and $\frac{b}{a}>2$, then $\left[\frac{a}{n}, \frac{b}{n}\right] \cap K \neq$ $\emptyset$ for every $n \in \mathbb{N}, n \geq 3 b$.

We take $N \in \mathbb{N}$ so that $3^{N} \leq \frac{n}{b}<3^{N+1}$ and then $\left(\frac{b}{n}\right) /\left(\frac{a}{n}\right)>2$ implies that the interval $\left(\frac{a}{n}, \frac{b}{n}\right)$ contains at least one of the points $\frac{1}{3^{N+1}}$ and $\frac{2}{3^{N+1}}$ of $C$.

In fact it is not very difficult to see that the one third Cantor set satisfies property (20) for every $t_{0} \in C$.

The question whether $C(\mathbb{T}) \cap U(C)=\emptyset$ is true is only slightly more complicated. The one third Cantor set $C \subseteq[0, \pi]$ is not symmetric with respect to 0 and in fact it is not symmetric with respect to any of its points. Nevertheless $C(\mathbb{T}) \cap U(C)=\emptyset$ is true.

Proposition 4.2. Let $K$ be a compact proper subset of $\mathbb{T}$ and $t_{0} \in K$ with the following property:

for each infinite $\mathbb{M} \subseteq \mathbb{N}$ there are $t_{m} \in K$ for all $m \in \mathbb{M}$

so that $t_{m} \rightarrow t_{0}$ and there are $a, b$ with $0<a<b<2 \pi$

and $[a / m, b / m] \cap\left(K-t_{m}\right) \cap\left(t_{m}-K\right) \neq \emptyset$

for infinitely many $m \in \mathbb{M}$.

If $f \in L^{1}(\mathbb{T}) \cap U(K)$, then the $(C, 1)$ means of the Fourier series of $f$ do not converge uniformly on $K$.

Proof. The proof is a variation of the proof of Proposition 4.1.

Let $S_{n}(f, t)=\sum_{k=-n}^{n} \widehat{f}(k) e^{i k t}$ be the partial sums of the Fourier series of $f$, and assume that their $(\mathrm{C}, 1)$ means converge to some function $s$ uniformly on $K$. We consider an arbitrary $h \in C(K)$ and a sequence $S_{n_{j}}(f, t)$ converging to $h$ uniformly on $K$.

Now there are $t_{j} \in K$ so that $t_{j} \rightarrow t_{0}$ and (considering a subsequence) corresponding $\theta_{j}$ so that $t_{j} \pm \theta_{j} \in K$ and $a \leq n_{j} \theta_{j} \leq b$ for all $j$.

We apply again Theorem (12.9) of [10], Vol I, Ch III and get

$$
\frac{1}{2}\left(S_{n_{j}}\left(f, t+\theta_{j}\right)+S_{n_{j}}\left(f, t-\theta_{j}\right)\right)-\left(S_{n_{j}}(f, t)-s(t)\right) \cos n_{j} \theta_{j} \rightarrow s(t)
$$

uniformly for $t \in K$. In fact here we apply the proof rather than the statement of the above Theorem (12.9) of [10]. One should read the small paragraph a few lines before the statement of Theorem (12.6): "we also observe that if the terms of $u_{0}+u_{1}+\ldots$ depend on a parameter, and if the hypotheses concerning this series are satisfied uniformly, the conclusions also hold uniformly". The relevant hypothesis in our case is the uniform $(\mathrm{C}, 1)$ summability of the Fourier series of $f$. 
Since $t_{j} \rightarrow t_{0}$ and $t_{j} \pm \theta_{j} \rightarrow t_{0}$ and since we may assume that $n_{j} \theta_{j} \rightarrow \phi$ for some $\phi \in[a, b]$, we finally get

$$
h\left(t_{0}\right)-\left(h\left(t_{0}\right)-s\right) e^{i \phi}=s
$$

and, choosing $h\left(t_{0}\right) \neq s$, we arrive at a contradiction.

Corollary 4.2. If $K$ is a compact proper subset of $\mathbb{T}$ which satifies property (21) for some $t_{0} \in K$ then $C(\mathbb{T}) \cap U(K)=\emptyset$.

In particular the one third Cantor set $C \subseteq[0, \pi]$ satisfies property (21) for every $t_{0} \in C$ and hence $C(\mathbb{T}) \cap U(C)=\emptyset$.

Proof. For the first statement we recall that if $f \in C(\mathbb{T})$ then the $(\mathrm{C}, 1)$ means of the Fourier series of $f$ converge to $f$ uniformly on $\mathbb{T}$.

The one third Cantor set satisfies property (21) in a slightly stronger form: there are $a, b$ with $0<a<b<2 \pi$ so that for every $t_{0} \in C$ there are $t_{n} \in C$ for all $n \in \mathbb{N}$ with $t_{n} \rightarrow t_{0}$ and $\left[\frac{a}{n}, \frac{b}{n}\right] \cap\left(K-t_{n}\right) \cap\left(t_{n}-K\right) \neq \emptyset$ for every $n \in \mathbb{N}$.

In fact, take arbitrary $a, b$ with $0<a<b<2 \pi$ and $\frac{b}{a}>3$ and any $t_{0} \in C$. For each $N \in \mathbb{N}$ we consider the interval $I_{N}$ of length $\frac{1}{3^{N}}$ which appears at the $N$-th step of the construction of $C$ and which contains $t_{0}$. If $I_{N,-}$ and $I_{N,+}$ are the left and right subintervals of $I_{N}$ of length $\frac{1}{3^{N+1}}$, then $t_{0}$ belongs to one of them (and this is $I_{N+1}$ ). If $t_{0} \in I_{N,-}$ then we define $t_{n}$ to be the right endpoint of $I_{N,-}$ for every $n$ with $3^{N} b \leq n<3^{N+1} b$. If $t_{0} \in I_{N,+}$ then we define $t_{n}$ to be the left endpoint of $I_{N,+}$ for every $n$ with $3^{N} b \leq n<3^{N+1} b$. In the case $t_{0} \in I_{N,-}$ the three points $t_{n}$ and $t_{n} \pm \frac{1}{3^{N+1}}$ belong to $C$ and $\frac{1}{3^{N+1}} \in\left(\frac{a}{n}, \frac{b}{n}\right)$ since $3^{N+1} a<3^{N} b \leq n<3^{N+1} b$. The case $t_{0} \in I_{N,+}$ is similar.

It is not hard to construct countably infinite sets satistfying (20). Take for example $K=\{0\} \cup\left\{\frac{1}{n} \mid n \in \mathbb{N}\right\}$. Hence,

Corollary 4.3. There are countably infinite compact $K \subseteq \mathbb{T}$ such that $A(\mathbb{D}) \cap U(K)=\emptyset$.

We close with the following open problems.

I. Construct a perfect set $K$ and a function $f \in A(\mathbb{D}) \cap U(K)$.

II. Study pointwise universality on uncountable sets $E$ with Lebesgue measure zero for functions in $C(\mathbb{T})$ or $A(\mathbb{D})$.

Of course the functions $g$ in Definition 1.1 of pointwise universality must belong to the first class of Baire.

III. Is it true that, if $E$ has positive Hausdorff dimension, then $E$ does not accept universality?

Acknowledgement. We would like to thank the referee for valuable comments which cleared certain subtle points of the proofs and 
helped us improve the presentation of this paper. Proposition 4.1 in our original paper contained nothing about sets $K$ symmetric with respect to $t_{0}$ and hence our original Corollary 4.1 considered only the result $A(\mathbb{D}) \cap U(C)=\emptyset$. The referee asked whether we could prove that $C(\mathbb{T}) \cap U(C)=\emptyset$ and this prompted us to work first with symmetric sets $K$ and get that $C(\mathbb{T}) \cap U\left(C^{*}\right)=\emptyset$. After a while we proved the stronger $C(\mathbb{T}) \cap U(C)=\emptyset$. We include the weaker result since it shows that we do not need extra hypotheses (i.e. the uniform $(\mathrm{C}, 1)$ summability) in the presence of the symmetry of the set $K$.

\section{REFERENCES}

[1] F. Bayart and Y. Heurteaux, Multifractal analysis for the divergence of Fourier series. arXiv:1101.3027

[2] G. Herzog and P. C. Kunstmann, Universally divergent Fourier series via Landau's extremal functions. Comment. Math. Univ. Carolinae 56 (2015), 159168.

[3] Y. Katznelson, Introduction to Harmonic Analysis. Cambridge Univ. Press, Cambridge, 3rd edition, 2004.

[4] J. P. Kahane, Baire's category theorem and trigonometric series. J. Anal. Math. 80 (2000), 143-182.

[5] E. Katsoprinakis, V. Nestoridis and C. Papachristodoulos, Universality and Cesaro summability. Comp. Methods and Function Theory 12 (2012), 419448.

[6] T. W. Körner, Kahane's Helson curve. Proccedings of the Conference in Honor of J. P. Kahane, Orsay, 1993, J. Fourier Anal. Appl. (1995), 325-346.

[7] J. Müller, Continuous functions with universally divergent Fourier series on small subsets of the circle. C. R. Acad. Sci. Paris, Ser. I 348 (2010), 1155-1158.

[8] V. Nestoridis, Universal Taylor series. Ann. Inst. Fourier 46, no 5 (1996), 1293-1306.

[9] J. Oxtoby, Measure and Category. Springer-Verlag Inc. New York, 2nd edition, 1980.

[10] A. Zygmund, Trigonometric Series, Vol. I. Cambridge Univ. Press, Cambridge, 3rd edition, 2002.

Department of Mathematics and Applied Mathematics, University of Crete, 70013 Iraklio, Crete, Greece

E-mail address: papadim@math.uoc.gr 